Gestationsdiabetes

\title{
Mit Screening und Gegenmaßnahmen mehr Lebensqualität und weniger Kosten
}

Lohse N, Marseille E, Kahn JG. Development of a model to assess the cost-effectiveness of gestational diabetes mellitus screening and lifestyle change for the prevention of type 2 diabetes mellitus. Int J Gynaecol Obstet 2011;115 Suppl 1:S20-5.
Fragestellung: Sind Frauen mit Gestationsdiabetes in der Anamnese eine gute Klientel für Maßnahmen zur Prävention von Typ-2-Diabetes?

Hintergrund: Zunehmend wird diskutiert, für welche Personen Maßnahmen zur Prävention von Typ-2-Diabetes am ehesten in Frage kommen und wie diese Personen effektiv gefunden werden können. Ein Gestationsdiabetes (GDM) ist ein entscheidender Risikofaktor für einen späteren Typ-2-Diabetes: Man geht davon aus, dass der GDM die Basis ist für bis zu 30\% der Neuerkrankungen. Das macht auch Sinn, da fast alle Frauen mit einem früheren GDM über die nächsten 10 bis 15 Jahre einen Typ-2-Diabetes entwickeln. Daher sind Frauen mit einem GDM eine attraktive Klientel, um frühzeitig mit Maßnahmen zu beginnen und somit den Ausbruch eines Diabetes zu verhindern. Es stellt sich auch die Frage, wie das kosteneffektiv geschehen kann.

Patienten und Methodik: Diese Studie hat sich mit der Entwicklung eines kosteneffektiven Modells zum Screening auf Gestationsdiabetes und nachfolgender Lebensstilintervention zur Diabetesprävention bei den Frauen mit GDM beschäftigt. Dieses Modell wurde in fünf Gesundheitszentren in Indien und Israel getestet. Zusätzlich wurde mit Hilfe eines mathematischen Modells der Zugewinn an Lebensjahren in hoher Lebensqualität ohne erholte Morbidität kalkuliert.

Ergebnisse: Ein umfassendes Screening von schwangeren Frauen auf GDM und einer Lebensstilintervention bei betroffenen Frauen post partum ergab einen Kostenspareffekt von 78 US-Dollar für Frauen mit GDM in Indien und 1945 US-Dollar für Frauen mit GDM in Israel. 2,33 zusätzliche Lebensjahre mit guter Lebens-

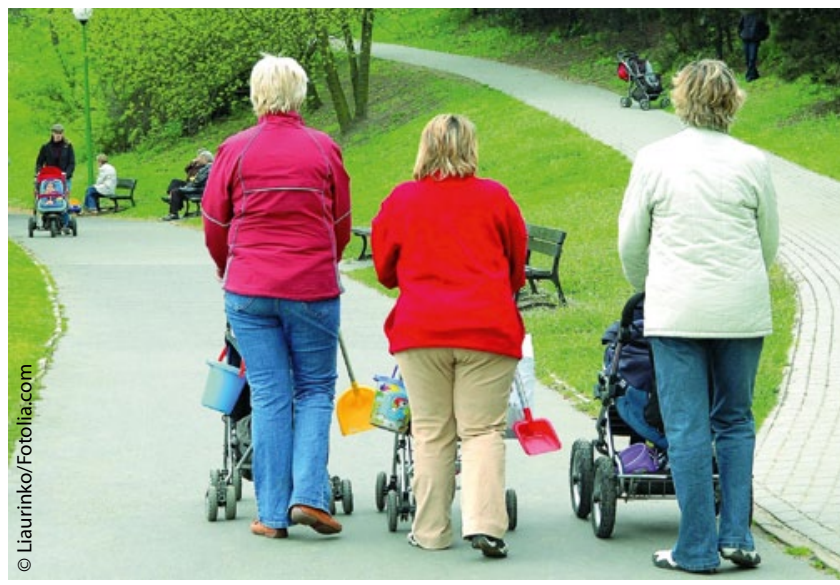

Wird bei Schwangeren ein Gestationsdiabetes (GDM) festgestellt und erfolgt auch post partum eine Lebensstilintervention zur Diabetesprävention, gelingt es offenbar, ihr Leben zu verlängern und Kosten zu sparen. Umso besser, dass Schwangere seit dem entprechenden Beschluss des GBA im Dezember 2011 künftig Anspruch auf einen GDM-Test als Leistung der gesetzlichen Krankenkassen haben. Der Beschluss basiert u.a. auf der Nutzenbewertung des IQWiG.

qualität wurden dadurch in Indien und 3,1 Jahre in Israel gewonnen. In Israel waren das Screening und die Intervention sogar kostensparend. In beiden Ländern war das Vorgehen effektiver als von der WHO für eine „hochkosteneffiziente Maßnahme“ definiert.

Schlussfolgerung: Ein GDM-Screening mit nachfolgender Lebensstilintervention in westlichen Gesundheitssystemen ist kostensparend und hat in sich entwickelnden Systemen eine attraktive Kosteneffizienz-Ratio.
Kommentar: Diese Studie ist interessant, da in Deutschland schon lange ein generelles Gestationsdiabetes-Screening diskutiert wird. Häufig wird es auch mit dem Kostenargument abgelehnt. Die Studie zeigt nun, dass in einem westlichen Gesundheitssystem ein GDM-Screening mit Lebensstilintervention zur Diabetesprävention kostensparend ist. Ein ähnliches Argument finden wir in der kürzlich publizierten Leitlinie des NICE, welche auch für Lebensstilmaßnahmen zur Diabetesprävention für Personen unter 37 Jahren ei- nen kostensparenden Effekt darlegt. Daher kann diese Studie als Landmark-Paper für die Analyse der Kosteneffizienz eines Screenings mit Diabetesprävention angesehen werden. Wir hoffen, dass sich solche Maßnahmen schneller als bisher in Deutschland umsetzen lassen.

Prof. Dr. med. Peter E. H. Schwarz, Dresden 